\title{
Planar Hall effect with sixfold oscillations in a Dirac antiperovskite
}

\author{
D. Huang $\odot,{ }^{1, *}$ H. Nakamura, ${ }^{2, \dagger}$ and H. Takagi ${ }^{1,3,4, \$}$ \\ ${ }^{1}$ Max Planck Institute for Solid State Research, 70569 Stuttgart, Germany \\ ${ }^{2}$ Department of Physics, University of Arkansas, Fayetteville, Arkansas 72701, USA \\ ${ }^{3}$ Institute for Functional Matter and Quantum Technologies, University of Stuttgart, 70569 Stuttgart, Germany \\ ${ }^{4}$ Department of Physics, University of Tokyo, 113-0033 Tokyo, Japan
}

(Received 10 November 2020; accepted 4 March 2021; published 22 March 2021)

\begin{abstract}
The planar Hall effect (PHE), wherein a rotating magnetic field in the plane of a sample induces oscillating transverse voltage, has recently garnered attention in a wide range of topological metals and insulators. The observed twofold oscillations in $\rho_{y x}$ as the magnetic field completes one rotation are the result of chiral, orbital, and/or spin effects. The antiperovskites $A_{3} B \mathrm{O}(A=\mathrm{Ca}, \mathrm{Sr}, \mathrm{Ba} ; B=\mathrm{Sn}, \mathrm{Pb})$ are topological crystalline insulators whose low-energy excitations are described by a generalized Dirac equation for fermions with total angular momentum $J=\frac{3}{2}$. We report unusual sixfold oscillations in the PHE of $\mathrm{Sr}_{3} \mathrm{SnO}$, which persisted nearly up to room temperature. Multiple harmonics (twofold, fourfold, and sixfold), which exhibited distinct field and temperature dependencies, were detected in $\rho_{x x}$ and $\rho_{y x}$. These observations are more diverse than those in other Dirac and Weyl semimetals and point to a richer interplay of microscopic processes underlying the PHE in the antiperovskites.
\end{abstract}

DOI: 10.1103/PhysRevResearch.3.013268

\section{INTRODUCTION}

The planar Hall effect (PHE), in contrast to the ordinary Hall effect (OHE), is defined as the transverse voltage that arises when an in-plane magnetic field is applied. Initially reported in ferromagnetic metals and semiconductors [1,2], the PHE has recently gained traction in a growing number of topological metals and insulators as a tool to probe their relativistic quasiparticles [3-21]. The interplay of chiral, orbital, and spin degrees of freedom that governs the PHE in these materials is rich and remains subject to active investigation.

At a phenomenological level, the PHE is driven by a magnetic-field-induced rotation of the principal axes of the resistivity tensor [19]. Suppose that an in-plane magnetic field $\mathbf{B}$ generates a resistive anisotropy whose principal axis $x^{\prime}$ is locked along the direction of $\mathbf{B}$ [Fig. 1(a)]. Then

$$
\left(\begin{array}{l}
\mathbf{E}_{x^{\prime}} \\
\mathbf{E}_{y^{\prime}}
\end{array}\right)=\left(\begin{array}{cc}
\rho_{\|} & 0 \\
0 & \rho_{\perp}
\end{array}\right)\left(\begin{array}{l}
\mathbf{i}_{x^{\prime}} \\
\mathbf{i}_{y^{\prime}}
\end{array}\right),
$$

where $\mathbf{E}$ is the electric field, $\mathbf{i}$ is the current density and $\rho_{\|}\left(\rho_{\perp}\right)$ is the magnetoresistivity when the current runs parallel (perpendicular) to $\mathbf{B}$. For an arbitrary in-plane angle $\phi$ between $\mathbf{B}$

\footnotetext{
*d.huang@fkf.mpg.de

†hnakamur@uark.edu

*h.takagi@fkf.mpg.de
}

Published by the American Physical Society under the terms of the Creative Commons Attribution 4.0 International license. Further distribution of this work must maintain attribution to the author(s) and the published article's title, journal citation, and DOI. Open access publication funded by the Max Planck Society. and $\mathbf{i}$, the resistivity tensor in the sample frame ( $x$ along $\mathbf{i})$ can be obtained from a coordinate transformation:

$$
\begin{aligned}
\left(\begin{array}{l}
\mathbf{E}_{x} \\
\mathbf{E}_{y}
\end{array}\right)= & \left(\begin{array}{cc}
\cos \phi & -\sin \phi \\
\sin \phi & \cos \phi
\end{array}\right)\left(\begin{array}{cc}
\rho_{\|} & 0 \\
0 & \rho_{\perp}
\end{array}\right) \\
& \times\left(\begin{array}{cc}
\cos \phi & \sin \phi \\
-\sin \phi & \cos \phi
\end{array}\right)\left(\begin{array}{l}
\mathbf{i}_{x} \\
\mathbf{i}_{y}
\end{array}\right) .
\end{aligned}
$$

By definition, $\mathbf{i}_{y}=0$, and we extract $\rho_{x x}$ and $\rho_{y x}$ :

$$
\begin{gathered}
\rho_{x x}=\frac{\left(\rho_{\perp}+\rho_{\|}\right)}{2}-\frac{\left(\rho_{\perp}-\rho_{\|}\right)}{2} \cos 2 \phi, \\
\rho_{y x}=-\frac{\left(\rho_{\perp}-\rho_{\|}\right)}{2} \sin 2 \phi .
\end{gathered}
$$

Here, $\rho_{x x}$ and $\rho_{y x}$ exhibit oscillations with two periods in a full rotation, $45^{\circ}$ relative phase shift, and equal amplitudes proportional to the difference between $\rho_{\perp}$ and $\rho_{\|}$[Fig. 1(b)]. By convention, Eq. (3) is called anisotropic magnetoresistance (AMR) and Eq. (4) is called the PHE. From the derivation, we observe that $\rho_{y x}$ arises as an off-diagonal element in the sample frame after the coordinate transformation from the magnetic-field frame. Unlike the case of the OHE for outof-plane fields, the resistivity tensor is symmetric, and the resulting PHE is even in field.

The situation is significantly enriched when we consider the additional crystalline anisotropy introduced by the underlying lattice. Equations (3) and (4) are no longer exact, and the lattice symmetry dictates which higher harmonics in $\rho_{x x}$ and $\rho_{y x}$ beyond the twofold oscillations are permitted. For example, in a crystal with an in-plane square lattice, up to a sixth-order expansion in $\phi$, twofold, fourfold, and sixfold oscillations are allowed in $\rho_{x x}$, whereas only twofold and sixfold oscillations are allowed in $\rho_{y x}$ [22]. Fourfold and sixfold 
(a)

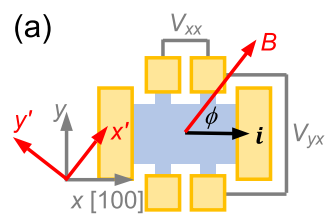

(c)

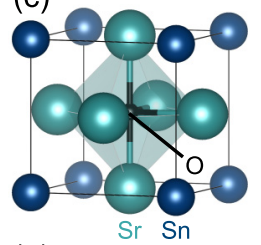

(e)
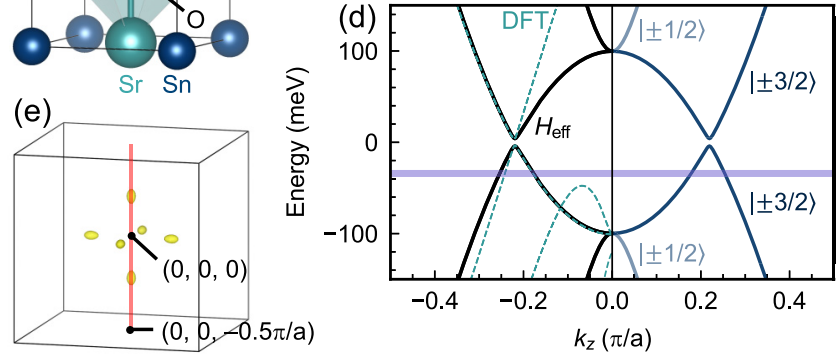

FIG. 1. (a) Device and measurement geometry. (b) Noncrystalline anisotropic magnetoresistance (AMR; $\rho_{x x}$ ) and planar Hall effect (PHE; $\rho_{y x}$ ) as predicted by Eqs. (3) and (4), assuming that $\rho_{\perp}>\rho_{\|}$. (c) Crystal structure of $\mathrm{Sr}_{3} \mathrm{SnO}$. (d) Approximate band structure as described by a $\mathbf{k} \cdot \mathbf{p}$ Hamiltonian $\left(H_{\text {eff }}\right)$. The bands along the $k_{z}$ axis are Kramers doublets and $j_{z}$ eigenstates $\left| \pm \frac{1}{2}\right\rangle$ and $\left| \pm \frac{3}{2}\right\rangle$ (right half of the plot). Bands calculated from density functional theory (DFT) are overlaid (left half of the plot). The purple line denotes the doping level corresponding to the carrier density of the measured films. (e) Fermi surface derived from $H_{\text {eff }}$. Only a portion of the Brillouin zone (BZ), where pockets exist, is shown. The red line denotes the cut along $k_{z}$ plotted in (d).

oscillations in $\rho_{x x}$ have been observed in a number of twodimensional (2D) oxide interfaces with square, orthorhombic, or hexagonal lattices [22-27]. However, absent of an obvious reason, the corresponding higher harmonics in $\rho_{y x}$ were not observed, including the sixfold oscillations purported to be allowed in a $2 \mathrm{D}$ square lattice.

The antiperovskites $A_{3} B \mathrm{O}(A=\mathrm{Ca}, \mathrm{Sr}, \mathrm{Ba} ; B=\mathrm{Sn}, \mathrm{Pb})$ are a unique family of compounds with unusual chemistry and electronic properties. Figure 1(c) depicts the crystal structure of $\mathrm{Sr}_{3} \mathrm{SnO}$, wherein the typical positions of the cations and anions in a perovskite are reversed. The low-energy spectrum can be described by a Dirac equation generalized to fermions with total angular momentum $J=\frac{3}{2}$ [28]:

$$
H_{\mathrm{eff}}(\mathbf{k})=m \tau_{z}+v_{1} \tau_{x} \mathbf{k} \cdot \mathbf{J}+v_{2} \tau_{x} \mathbf{k} \cdot \tilde{\mathbf{J}} .
$$

Here, $\boldsymbol{\tau}$ is the set of Pauli matrices with $\tau_{z}= \pm 1$ labeling the orbitals of the valence and conduction bands, $\mathbf{J}$ is the set of spin- $\frac{3}{2}$ matrices and $\tilde{\mathbf{J}}$ is a linear combination of $\mathbf{J}$ and $\mathbf{J}^{3}$ that shares the same transformation properties as $\mathbf{k}$ under the cubic point group. Equation (5) describes the inversion of two $J=$ $\frac{3}{2}$ quartets near the Brillouin zone (BZ) center, resulting in three-dimensional (3D), slightly gapped Dirac nodes along six equivalent $\Gamma-X$ cuts [29-31] [Figs. 1(d) and 1(e)]. Microscopically, these states are composed of $\mathrm{Sr} 4 d$ and $\mathrm{Sn} 5 p$ orbitals. The latter orbitals experience strong spin-orbit coupling and come in the linear combinations $-\left(\left|p_{x} \uparrow\right\rangle+i \mid p_{y} \uparrow\right) / \sqrt{2}$ and $\left(\left|p_{x} \downarrow\right\rangle-i \mid p_{y} \downarrow\right) / \sqrt{2}$, where $\uparrow$ and $\downarrow$ denote the spin, cor-

responding to the eigenstates $\left|j_{z}=+\frac{3}{2}\right\rangle$ and $\left|j_{z}=-\frac{3}{2}\right\rangle$ [29]. $\mathrm{Sr}_{3} \mathrm{SnO}$ is also a candidate topological crystalline insulator hosting type I and II Dirac surface states [28,32], as well as a higher-order topological insulator hosting hinge states under broken symmetry [33].

Previous experiments of $\mathrm{Sr}_{3} \mathrm{SnO}$ have probed its 3D Dirac carriers [34-36], observed superconductivity upon hole doping [37] (with possible implications for higher spin Cooper pairing [38]), and detected weak antilocalization (WAL) due to spin-orbital entanglement [39]. Here, we measured the inplane-field magnetoresistance of thin films of $\mathrm{Sr}_{3} \mathrm{SnO}(001)$ grown by molecular beam epitaxy (MBE) and found them to be a candidate system with an in-plane square lattice exhibiting sixfold oscillations in the PHE. More specifically, the AMR showed twofold and fourfold oscillations, while the PHE showed twofold and sixfold oscillations. Remarkably, the sixfold oscillations persisted up to the highest temperature measured $(T=250 \mathrm{~K})$. The different harmonics in the AMR and PHE also behaved distinctly with respect to magnetic field and temperature, pointing to a complex interplay of underlying microscopic mechanisms. We speculate on the possible role of the spin-orbital-entangled $J=\frac{3}{2}$ fermions in some of these observations.

\section{METHODS}

Thin films of $\mathrm{Sr}_{3} \mathrm{SnO}$ were deposited on yttria-stabilized $\mathrm{ZrO}_{2}$ in an Eiko MBE chamber with base pressure in the range of mid- $10^{-10}$ Torr. Detailed growth procedures, as well as film characterization via $x$-ray diffraction (XRD), low-energy electron diffraction, and x-ray photoelectron spectroscopy, can be found in Refs. [39,40].

Due to the extreme chemical reactivity of the antiperovskites, postdeposition lithography of the films is challenging. Instead, during deposition, we used sapphire masks with millimeter-sized cutouts shaped as Hall bars to obtain films with well-defined geometries (width $W=2 \mathrm{~mm}$, aspect ratio $W / L=2$ ). For each deposition, four substrates were used, since each sample could only be used for one measurement. After the MBE growth, the films were transported via a vacuum suitcase to an Ar glove box furnished with a metal evaporator. The films dedicated to thickness calibration (Dektak stylus profilometer) or XRD were covered with Au to prevent degradation in ambient environment. For the films dedicated to transport, we evaporated Au contact pads, covered the films with grease (Apiezon N), and attached them to rotation sample pucks using $\mathrm{Au}$ wires and $\mathrm{Ag}$ epoxy (EPOTEK E4110, low-temperature cure), all within the glove box. Two distinct pucks were used to allow in-plane and out-ofplane rotation in a magnetic field. The films were then rapidly transported to a physical property measurement system with a $14 \mathrm{~T}$ magnet (Quantum Design). The base temperature for the rotation insert was $1.8 \mathrm{~K}$, and the highest temperature that could be measured before the antiperovskite films showed signs of degradation was $250 \mathrm{~K}$.

Our Eiko MBE chamber is attached to a customized Unisoku scanning tunneling microscope (STM) that operates at room temperature, with base pressure in the range of mid$10^{-10}$ Torr. Films for imaging were transferred in situ from the MBE to the STM. W tips were sharpened via electron-beam 

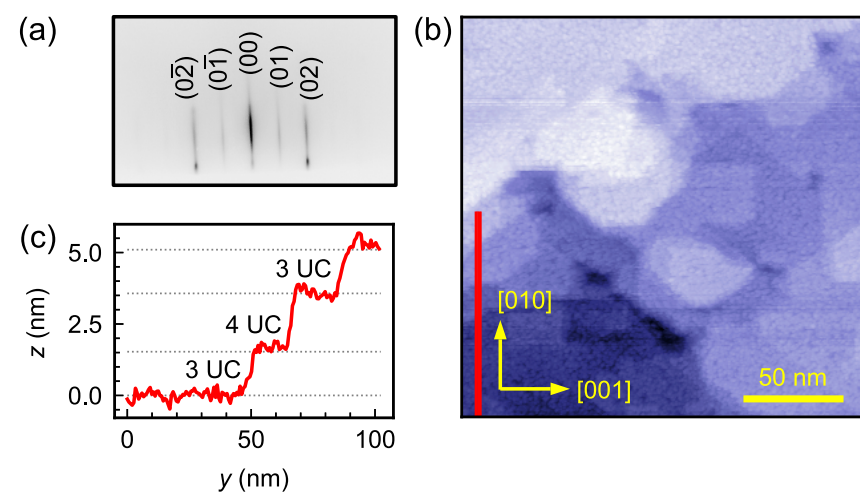

FIG. 2. (a) Reflection high-energy electron diffraction (RHEED) image of $\mathrm{Sr}_{3} \mathrm{SnO}(001)$ taken along the [100] direction (sample SS121). Electron energy: $15 \mathrm{keV}$. (b) Scanning tunneling microscope (STM) topography acquired at room temperature. Set point: $-1.75 \mathrm{~V}$, $10 \mathrm{pA}$. (c) Averaged line cut along the vertical red line in (b), which has a width of 5 pixels. The steps correspond to integer multiples of the unit cell (UC; approximately $0.51 \mathrm{~nm}$ ), as labeled in the plot.

bombardment. Atomic-resolution imaging was confirmed on highly oriented pyrolitic graphite.

The density functional theory (DFT) bands shown in Fig. 1(d) were reproduced from Ref. [40]. Detailed parameters can be found there. The doping level corresponding to the carrier density of the films was determined by integrating the density of states. Measured concentrations of $1.9-2.6 \times 10^{19}$ holes $/ \mathrm{cm}^{3}$ correspond to a doping level of $30 \mathrm{meV}$ below the valence band maximum.

Following Ref. [28], we made a substitution to the mass term in $H_{\text {eff }}$ [Eq. (5)]: $m \rightarrow m+\alpha k^{2}$. This was deemed necessary to properly classify the topology of the system. Parameters used to reproduce the DFT band structure are $m=$ $-0.09975 \mathrm{eV}, v_{1}=0.134 \mathrm{eV}, v_{2}=0.39 \mathrm{eV}$, and $\alpha=0.21 \mathrm{eV}$. These values result in a mirror Chern number of $n_{M}=-2$, although identical band dispersions with $n_{M}=+2$ are also possible with different choices of $v_{1}$ and $v_{2}$.

The crystal structure in Fig. 1(c) and the isosurface in Fig. 1(e) were visualized using VESTA [41].

\section{RESULTS}

\section{A. Surface characterization}

Figure 2(a) shows reflection high-energy electron diffraction (RHEED) patterns detected after film deposition (sample SS121), exhibiting streaks corresponding to a $1 \times 1$ lattice of $\mathrm{Sr}_{3} \mathrm{SnO}(001)$. The same film was then imaged at room temperature via STM under ultra-high vacuum [Fig. 2(b)]. We observed islands with edges oriented preferably along the [110] and [110] crystal axes, and sometimes along [100] and [010], attesting to the epitaxial growth of the film. Line-cut analysis [Fig. 2(c)] reveals step edges with heights corresponding to integer multiples of the unit cell (UC), roughly $0.51 \mathrm{~nm}$. We were unable to resolve an atomic lattice, likely due to complications arising from surface polarity and valence instability [40].
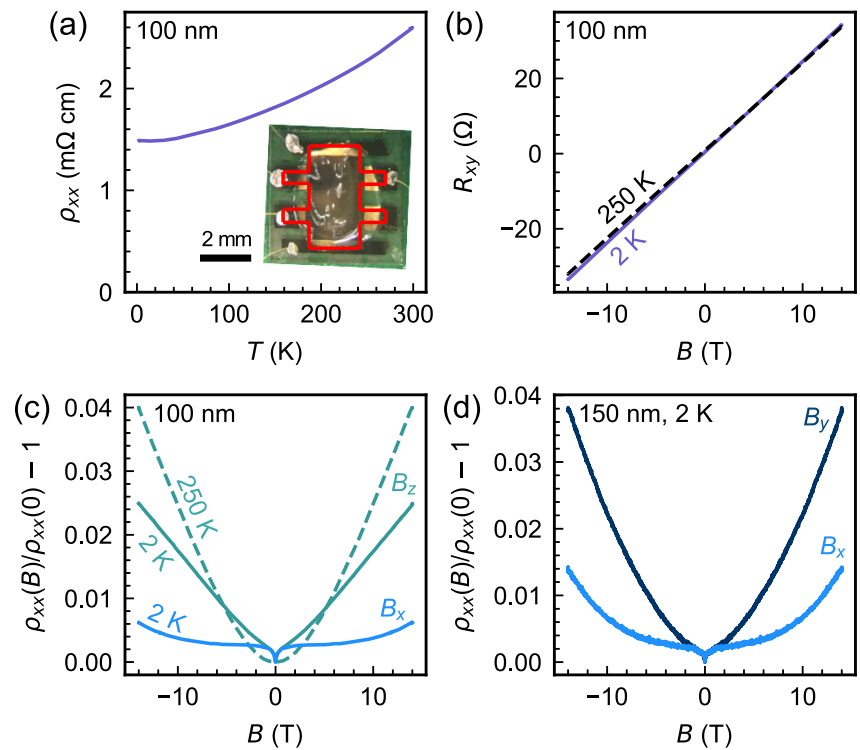

FIG. 3. (a) Longitudinal resistivity measured for a 100-nm-thick film (SS124) from room temperature down to $2 \mathrm{~K}$. Inset: optical micrograph of the Hall-bar device. (b) Ordinary Hall effect (OHE) due to an out-of-plane magnetic field, measured at 2 and $250 \mathrm{~K}$. (c) Magnetoresistivity at 2 and $250 \mathrm{~K}$ for an out-of-plane field $\left(B_{z}\right)$ and at $2 \mathrm{~K}$ for an in-plane field parallel to the current $\left(B_{x}\right)$. (d) In-plane-field magnetoresistivity at $2 \mathrm{~K}$ for a 150 -nm-thick film (SS123). The magnetoresistivities measured under $B_{x}$ and $B_{y}$ correspond to $\rho_{\|}$and $\rho_{\perp}$, respectively. The traces in (c) and (d) are symmetrized with respect to field.

\section{B. Transport}

Figure 3(a) presents the temperature-dependent resistivity $\rho_{x x}$ of a 100 -nm-thick film (SS124), which manifests semimetallic behavior. The residual resistivity ratio (RRR), defined using data points at $T=297$ and $2 \mathrm{~K}$, is roughly 1.7. We note that the temperature dependence and RRR are very similar to $\mathrm{Sr}_{3} \mathrm{SnO}$ films measured using in situ four-probe transport [36]; therefore, we conclude that no significant degradation was induced by capping our films with grease.

Figure 3(b) displays OHE measurements in an out-of-plane field at $T=2$ and $250 \mathrm{~K}$. Here, $R_{x y}$ is linear and has a positive slope corresponding to hole conduction, without any hints of mixed carriers. Minimal temperature dependence is observed. The extracted hole density and mobility at $2 \mathrm{~K}$ are $2.6 \times 10^{19} \mathrm{~cm}^{-3}$ and $162 \mathrm{~cm}^{2} /(\mathrm{Vs})$. The corresponding Fermi surface comprises the six Dirac pockets shown in Fig. 1(e), as well as an additional set of pockets along $\Gamma-M$ not captured by the effective model [39]. The parameters of all devices reported in this paper are summarized in Table I.

The magnetoresistivity in an out-of-plane field is shown in Fig. 3(c). At $250 \mathrm{~K}, \rho_{x x}$ increases by a few percent at $14 \mathrm{~T}$ and displays a $B^{2}$ dependence characteristic of an orbital effect; however, our subsequent discussion will caution against such a straightforward interpretation. At $2 \mathrm{~K}, \rho_{x x}$ shows a dip due to WAL below fields of $1 \mathrm{~T}$. At higher fields, $\rho_{x x}$ appears to scale linearly with field, but we note that we did not consistently observe this behavior across all samples. Interestingly, 
TABLE I. Samples measured. The carrier densities were extracted from the OHE at $2 \mathrm{~K}$, which then yielded the corresponding mobilities.

\begin{tabular}{llcccc}
\hline \hline Sample & Figures & $\begin{array}{c}\text { Thickness } \\
(\mathrm{nm})\end{array}$ & $\begin{array}{c}\text { Carrier density } \\
\left(10^{19} \text { holes } / \mathrm{cm}^{3}\right)\end{array}$ & $\begin{array}{c}\text { Mobility } \\
{\left[\mathrm{cm}^{2} /(\mathrm{Vs})\right]}\end{array}$ \\
\hline SS114 & 5,6 & 200 & 2.4 & 343 & Measurement \\
SS119 & 5,6 & 50 & 1.9 & - & Transport \\
SS121 & 2 & 100 & - & 92 & Transport \\
SS123 & $3,5,6$ & 150 & 2.6 & 162 & Transport \\
SS124 & $3,4,5,6$ & 100 & Transport \\
\hline \hline
\end{tabular}

the percentage change in $\rho_{x x}$ at $14 \mathrm{~T}$ is smaller at $2 \mathrm{~K}$ than at $250 \mathrm{~K}$. Also shown in Fig. 3(c) is the magnetoresistivity trace at $2 \mathrm{~K}$ for an in-plane field, aligned along the current direction. The WAL dip remains identical, but the high-field magnetoresistivity is moderately reduced. The isotropy of the WAL attests to the film being in an electronically 3D regime; further support can be found in Ref. [39].

Since AMR and the PHE involve rotations of the magnetic field in the plane of the sample, we show the in-plane-field magnetoresistivity of another 150-nm-thick sample (SS123) at $2 \mathrm{~K}$ [Fig. 3(d)]. The in-plane field is applied parallel and perpendicular to the current, corresponding to $\rho_{\|}$and $\rho_{\perp}$ in Eqs. (3) and (4). Again, the WAL dip remains identical, but $\rho_{\perp}>\rho_{\|}$at higher fields.

\section{AMR and PHE}

We acquired $\rho_{x x}$ (AMR) and $\rho_{y x}$ (PHE) for the 100nm-thick film (SS124) while applying a current along the [100] direction and rotating the device in a fixed, in-plane magnetic field of $14 \mathrm{~T}$. Here, $\phi$ is defined relative to the [100] axis. Measurements at 2 and $250 \mathrm{~K}$ are displayed in
Figs. 4(a)-4(d). Due to wobbling in the sample puck during rotation, the device experienced a small out-of-plane component of $\mathbf{B}$ with changing magnitude. In the $\rho_{y x}$ channel, an out-of-plane field generates an additional contribution from the OHE, which is odd in field. This spurious contribution can be cleanly removed by symmetrizing $\rho_{y x}$ acquired at +14 and -14 T [Figs. 4(b) and 4(d)], since the PHE itself is even in field [42]. On the other hand, as seen in Figs. 4(a) and 4(c), $\rho_{x x}$ is nearly identical for +14 and $-14 \mathrm{~T}$.

Qualitatively, the oscillations in $\rho_{x x}$ largely obey the $\cos 2 \phi$ dependence of Eq. (3). Maxima appear at $\phi=90^{\circ}$ and $270^{\circ}$, and minima appear at $\phi=180^{\circ}$ and $360^{\circ}$. The negative amplitude indicates that $\rho_{\perp}>\rho_{\|}$. Closer inspection of Figs. 4(a) and $4(\mathrm{c})$ reveals an asymmetry between the maxima, which have a broader width, and the minima, which have a narrower width, hinting at higher harmonics. The situation is significantly more pronounced in the $\rho_{y x}$ channel. At $2 \mathrm{~K}$ [Fig. 4(b)], maxima appear at $\phi=135^{\circ}$ and $315^{\circ}$, and minima appear at $\phi=225^{\circ}$ and $405^{\circ}$, consistent with a negative $\sin 2 \phi$ form. However, higher harmonics are visibly superimposed. At $250 \mathrm{~K}$ [Fig. 4(d)], the oscillations in $\rho_{y x}$ become predominantly sixfold, with maxima spaced $60^{\circ}$ apart.
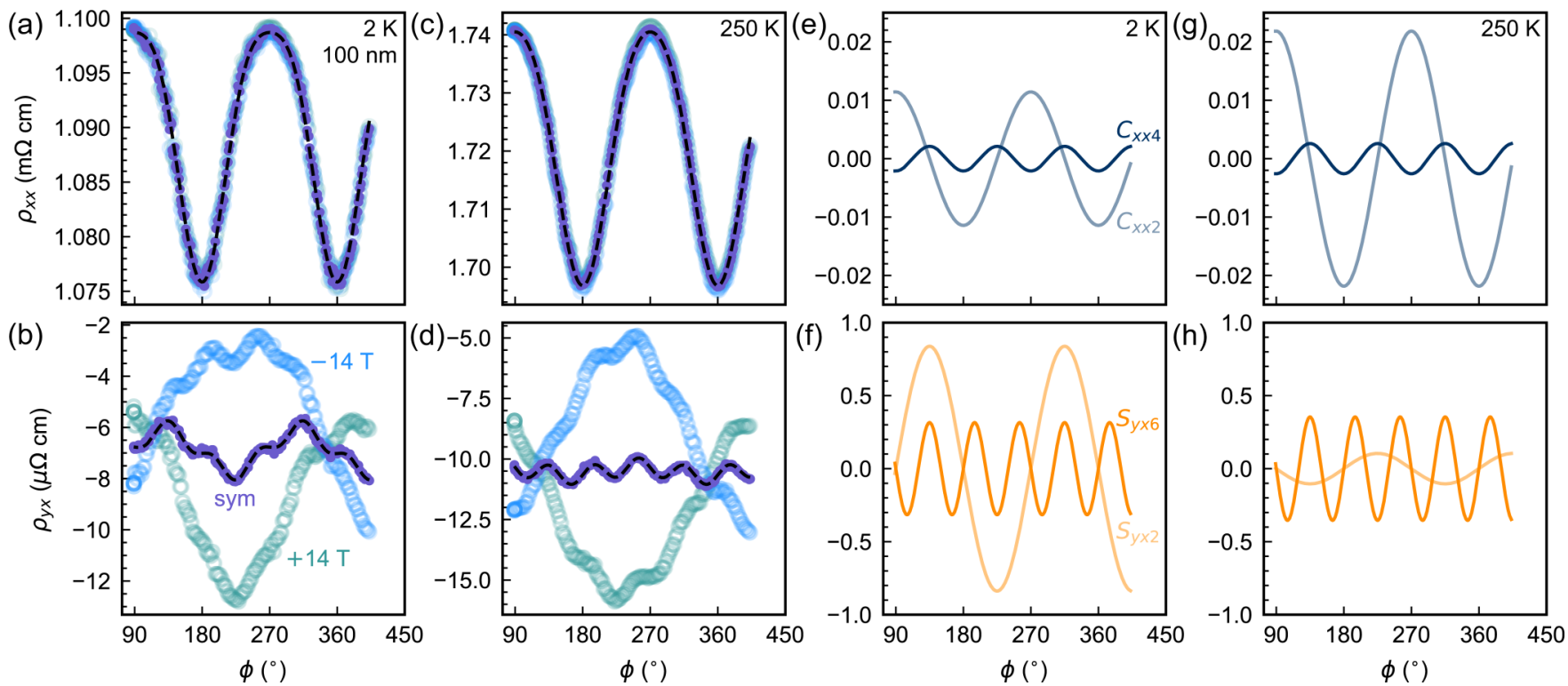

FIG. 4. (a) and (b) Anisotropic magnetoresistance (AMR; $\rho_{x x}$ ) and planar Hall effect (PHE; $\rho_{y x}$ ) at $T=2 \mathrm{~K}$. The open circles denote the raw data acquired at $B= \pm 14 \mathrm{~T}$, while the filled circles denote the field-symmetrized data. The overlaid dashed lines are fits to Eqs. (6) and (7) in the text. (c) and (d) AMR and PHE at $T=250 \mathrm{~K}$. (e)-(h) Decomposition of the fits in (a)-(d), respectively, to various harmonics. Film thickness is $100 \mathrm{~nm}$ (SS124). 

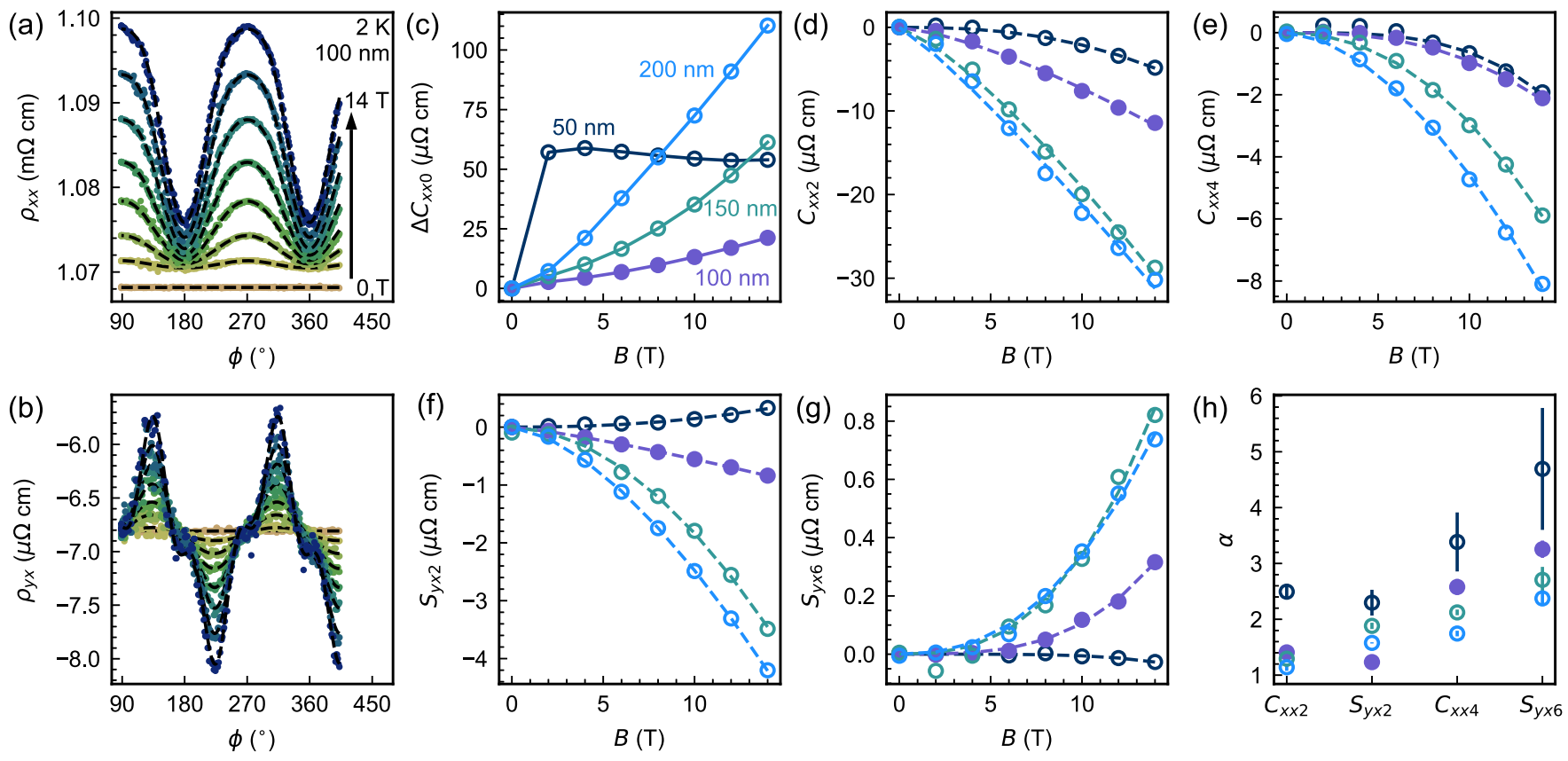

FIG. 5. (a) and (b) $\rho_{x x}$ and $\rho_{y x}$ acquired in $2 \mathrm{~T}$ intervals from 0 to $14 \mathrm{~T}$ (direction of arrow indicates increasing field). Fits to Eqs. (6) and (7) are overlaid as dashed lines. $T=2 \mathrm{~K}$, film thickness is $100 \mathrm{~nm}$ (SS124). (c)-(g) Field dependencies of the $C_{x x 0}, C_{x x 2}, C_{x x 4}, S_{y x 2}$, and $S_{y x 6}$ amplitudes extracted from (a) and (b) (filled circles). Data from three additional films with different thicknesses (SS114, SS119, SS123) are also plotted (open circles). In (c), $\Delta C_{x x 0}=C_{x x 0}(B)-C_{x x 0}(0)$. In (d)-(g), fits to power laws of the form $B^{\alpha}$ are overlaid as dashed lines. (h) Fit values of $\alpha$ vs harmonic for the different films.

Motivated by the allowed harmonics for a 2D square lattice [22], we employed the following minimal model to fit the observed AMR and PHE:

$$
\begin{gathered}
\rho_{x x}=C_{x x 0}+C_{x x 2} \cos 2 \phi+C_{x x 4} \cos 4 \phi, \\
\rho_{y x}=S_{y x 2} \sin 2 \phi+S_{y x 6} \sin 6 \phi .
\end{gathered}
$$

Figures 4(e)-4(h) show the extracted twofold and fourfold oscillations in $\rho_{x x}$ and twofold and sixfold oscillations in $\rho_{y x}$. We observe that $C_{x x 2}$ and $S_{y x 2}$ have different magnitudes at 2 and $250 \mathrm{~K}$, but $C_{x x 4}$ and $S_{y x 6}$ are constant. Due to small random misalignments in the transverse voltage contacts, there is some admixture of $\rho_{x x}$ in $\rho_{y x}$. Hence, we supplement Eq. (7) with the terms $C_{y x 0}$ and $C_{y x 2} \cos 2 \phi$ to fit $\rho_{y x}$, but they are not intrinsic to the PHE.

\section{Field, temperature and thickness dependencies}

We next examined the field and temperature dependencies of the AMR and PHE for a total of four films with similar hole concentrations but varying thicknesses of $50 \mathrm{~nm}$ (SS119), $100 \mathrm{~nm}$ (SS124), $150 \mathrm{~nm}$ (SS123), and $200 \mathrm{~nm}$ (SS114). Figures 5(a) and 5(b) track the evolution of the AMR and PHE at $2 \mathrm{~K}$ as the field is increased from 0 to $14 \mathrm{~T}$. Again, the data were symmetrized with respect to field. The corresponding evolutions of $C_{x x 0}, C_{x x 2}, C_{x x 4}, S_{y x 2}$, and $S_{y x 6}$ are plotted in Figs. 5(c) $-5(\mathrm{~g})$. The $C_{x x 0}$ term corresponds to the average of $\rho_{\perp}$ and $\rho_{\|}$. A dip at zero field due to WAL is present at $2 \mathrm{~K}$ and especially pronounced in the thinnest film of $50 \mathrm{~nm}$. To aid comparison across the samples, we plot the change in $C_{x x 0}$, defined as $\Delta C_{x x 0}=C_{x x 0}(B)-C_{x x 0}(0)$, in Fig. 5(c). Since the WAL dip is identical for $\rho_{\perp}$ and $\rho_{\|}$[Fig. 3(d)], it does not factor into the higher harmonics. In fact, the higher harmonics all obey some power-law dependence of the form $B^{\alpha}$. The extracted values of the exponent $\alpha$ are presented in Fig. 5(h). We note that the error bar is rather large for $\alpha\left(S_{y x 6}\right)$ in the 50-nm-thick film, due to the smallness of $S_{y x 6}$. Despite the overall variation in $\alpha$, two trends are discernible: $\alpha$ increases as the harmonic increases and as the film thickness decreases.

Figures 6(a)-6(e) track the evolutions of $C_{x x 0}, C_{x x 2}$, $C_{x x 4}, S_{y x 2}$, and $S_{y x 6}$ as the temperature is raised from 2 to $250 \mathrm{~K}$. The $C_{x x 0}$ term simply mimics the temperature dependence of $\rho_{x x}$ at zero field [Fig. 3(a)]. With respect to the higher harmonics, however, we observe an unusual dichotomy between $C_{x x 2}$ and $S_{y x 2}$, which show clear temperature dependence, and $C_{x x 4}$ and $S_{y x 6}$, which are relatively independent of temperature up to $250 \mathrm{~K}$. However, even among the temperature-dependent harmonics, $C_{x x 2}$ and $S_{y x 2}$ behave dissimilarly: $C_{x x 2}$ increases while $S_{y x 2}$ decreases with increasing temperature. Thus, we observe harmonics that increase, decrease, and remain constant with respect to temperature. This diversity is unprecedented and defies the usual expectations for semiclassical magnetoresistance, whose amplitude scales with the carrier mobility. As seen in Fig. 6(f), the hole mobility decreases with increasing temperature, a trend that is only shared by one of the harmonics, $S_{y x 2}$.

From Figs. 5 and 6, we observe that, in general, the amplitudes of the harmonics increase as the film thickness increases. However, the 50-nm-thick sample (SS119) is anomalous in several regards. The sign of $S_{y x 2}$ is flipped from negative to positive, its amplitude grows with increasing temperature, and $S_{y x 6}$ is nearly suppressed below $100 \mathrm{~K}$. Our previous WAL measurements established that $50-\mathrm{nm}$ thick samples lie in a quasi-2D electronic regime, based on 

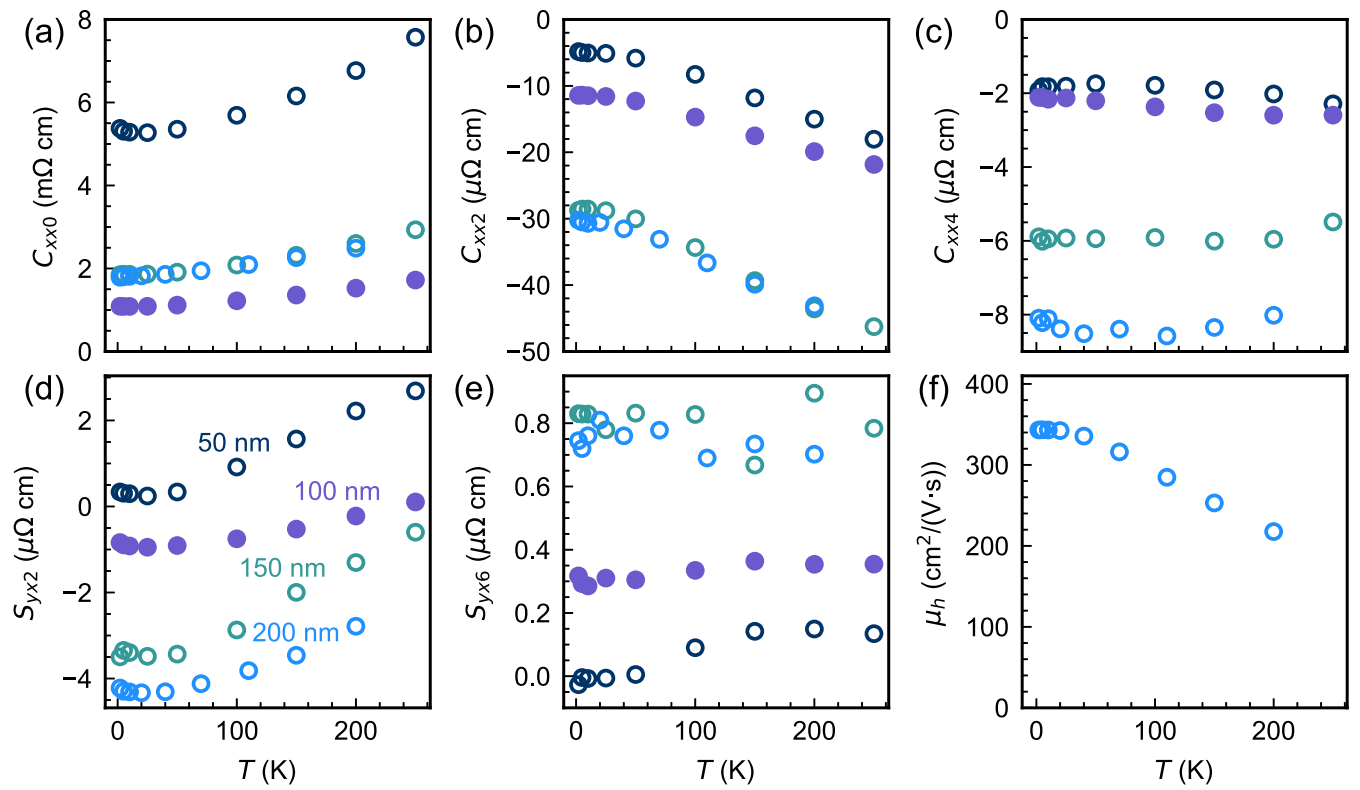

FIG. 6. (a)-(e) Temperature dependencies of the $C_{x x 0}, C_{x x 2}, C_{x x 4}, S_{y x 2}$, and $S_{y x 6}$ amplitudes. Data from a total of four films with different thicknesses are shown. (f) Hole mobility corresponding to the 200-nm-thick film (SS114).

the temperature dependence of the phase coherence length [39]. The changes in the PHE here could reflect such a 3D-2D crossover or also the lower mobility of this thinnest sample.

\section{DISCUSSION}

To summarize, the existence of sixfold oscillations in the PHE and the manifold behaviors of the different harmonics are distinguishing features of our experiment on $\mathrm{Sr}_{3} \mathrm{SnO}$. We emphasize that the in-plane symmetry of $\mathrm{Sr}_{3} \mathrm{SnO}(001)$ is fourfold, and neither RHEED nor STM uncovered any signatures of sixfold structure. According to a symmetry analysis by Rout et al. [22], the most general expansions of the AMR and PHE for a 2D square lattice are

$$
\begin{aligned}
\rho_{x x}= & a_{0}+a_{2} \cos 2 \phi_{B} \cos 2 \phi_{i}+b_{2} \sin 2 \phi_{B} \sin 2 \phi_{i} \\
& +a_{4} \cos 4 \phi_{B}+a_{6} \cos 6 \phi_{B} \cos 2 \phi_{i} \\
& +b_{6} \sin 6 \phi_{B} \sin 2 \phi_{i}+\cdots,
\end{aligned}
$$

and

$$
\begin{aligned}
\rho_{y x}= & -a_{2} \cos 2 \phi_{B} \sin 2 \phi_{i}+b_{2} \sin 2 \phi_{B} \cos 2 \phi_{i} \\
& -a_{6} \cos 6 \phi_{B} \sin 2 \phi_{i}+b_{6} \sin 6 \phi_{B} \cos 2 \phi_{i}+\cdots,
\end{aligned}
$$

where $\phi_{B}\left(\phi_{i}\right)$ is the angle between the magnetic field (current) and a reference crystallographic axis. In our case, the reference axis is [100], $\phi_{B}=\phi$ and $\phi_{i}=0$, yielding

$$
\rho_{x x}=a_{0}+a_{2} \cos 2 \phi+a_{4} \cos 4 \phi+a_{6} \cos 6 \phi+\cdots,
$$

and

$$
\rho_{y x}=b_{2} \sin 2 \phi+b_{6} \sin 6 \phi+\cdots .
$$

Our fitting model [Eqs. (6) and (7)] thus respects the terms permitted in a 2D square lattice. From the analysis, we also conclude that the existence of $C_{x x 4}$ but absence of $S_{y x 4}$ is a direct consequence of the lattice symmetry. We note that
Eqs. (10) and (11) enforce no constraints on the relative magnitudes of the harmonics. In our data, $S_{y x 6}$ is clearly visible, but $C_{x x 6}$ is somehow too small to extract.

We now consider each harmonic in greater detail. To start off, $C_{x x 2}$ is negative in amplitude, implying that $\rho_{\perp}>\rho_{\|}$, based on Eq. (3). Similar $C_{x x 2}$ with negative amplitude was observed in other Dirac and Weyl semimetals and ascribed to the chiral anomaly, orbital magnetoresistance, or a combination of the two [5-17]. In the chiral anomaly, the magnetoresistivity becomes negative when $\mathbf{B}$ and $\mathbf{i}$ are aligned, leading to $\rho_{\perp}>\rho_{\|}$. In orbital magnetoresistance, the Lorentz force is maximized when $\mathbf{B}$ and $\mathbf{i}$ are perpendicular, leading also to $\rho_{\perp}>\rho_{\|}$. The absence of negative longitudinal magnetoresistivity in Figs. 3(c) and 3(d) clearly favors the latter scenario. However, the temperature dependence of $C_{x x 2}$ is inconsistent with a pure orbital effect. Rather than scaling with the carrier mobility and increasing at low temperatures, it decreases at low temperatures [Fig. 6(b)], like the magnetoresistivity in Fig. 3(c). This anomalous temperature dependence may point to a competition between orbital magnetoresistance generating negative $C_{x x 2}$ and another process generating positive $C_{x x 2}$ $\left(\rho_{\|}>\rho_{\perp}\right)$. From Fig. 6(b), we deduce that orbital magnetoresistance dominates, resulting in an overall negative sign for $C_{x x 2}$, but that the competing process with positive $C_{x x 2}$ grows at low temperatures, such that the overall magnitude of $C_{x x 2}$ is reduced.

A similar behavior in $C_{x x 2}$ was reported in $\mathrm{SmB}_{6}$, where the sign of the AMR and PHE flipped from negative to positive at low temperatures $[20,21]$. This observation was attributed to the competition between orbital magnetoresistance in the bulk and anisotropic scattering of spin-polarized surface states. The latter produces positive $C_{x x 2}$ [19], but only when the sample is rotated in the plane of the magnetic field, due to some mechanisms still under debate involving scattering off magnetic impurities or band warping by the Zeeman effect [43]. To test for the possible influence of surface states, arising 
either from nontrivial topology or a surface reconstruction, we performed AMR measurements while rotating a sample out of the plane of the magnetic field [42]. If surface states were responsible for the anomalous temperature dependence of $C_{x x 2}$ in an in-plane field, then a different temperature dependence of $C_{x x 2}$ in an out-of-plane field would be expected. The reason is that the positive $C_{x x 2}$ contribution from surface states would vanish in an out-of-plane field, resulting in pure orbital magnetoresistance with negative $C_{x x 2}$ whose amplitude grows at low temperatures. We found that $C_{x x 2}$ behaved identically for in-plane and out-of-plane fields, with similar magnitudes and the same anomalous temperature dependence. We surmise that the competing processes must be bulk in nature, a point which we revisit.

The sizable difference between the magnitudes of $C_{x x 2}$ and $S_{y x 2}$ and their contrasting temperature dependencies is another puzzle for which we presently lack a microscopic picture. On a phenomenological level, the much smaller amplitude of $S_{y x 2}$ implies that $C_{x x 2}$ has a sizable crystalline contribution [44]; i.e., there is a $\rho_{x x}$ contribution which modulates with the angle between $\mathbf{B}$ and some crystal axis, rather than the angle between $\mathbf{B}$ and $\mathbf{i}$. In this case, the principal axes of the resistivity tensor do not rotate with the magnetic field, but remain locked to the sample, such that $\rho_{y x}$, which is an off-diagonal element of the resistivity tensor in the sample frame, does not appear. The sign change in $S_{y x 2}$ for the thinnest sample is another mystery. Perhaps the band structure is modified in the 2D limit, or the PHE is sensitive to the disorder potential, given that the 50-nm-thick film has the lowest mobility.

The fact that $C_{x x 4}$ and $S_{y x 6}$ are both relatively independent of temperature up to $250 \mathrm{~K}$ suggests that the two are linked in nature. The origin of their temperature independence is mysterious, but we mention in passing that, phenomenologically, it is somewhat reminiscent of the linear magnetoresistance in nonstoichiometric silver chalcogenides [45], as well as graphene [46] and $\mathrm{Bi}_{2} \mathrm{Te}_{3}$ [47], which shows weak temperature dependence up to room temperature.

Finally, it is instructive to ask what distinguishes the Dirac antiperovskite $\mathrm{Sr}_{3} \mathrm{SnO}$ from other 3D Dirac and Weyl semimetals, where simpler AMR and PHE were consistently reported [5-17]. In general, the higher harmonics previously observed in $\rho_{x x}$ were associated with 2D systems [22-27]. (One exception is $\mathrm{Bi}(111)$, which showed fourfold oscillations in $\rho_{y x}$ due to its highly anisotropic pockets [18].) We consider the bulk spin-orbital-entangled states of $\mathrm{Sr}_{3} \mathrm{SnO}$, which at low energies may be described as $J=\frac{3}{2}$ Dirac fermions. Theoretical work by Trushin et al. [48] demonstrated that AMR with positive $C_{x x 2}$ is possible within a 3D, spherical Kohn-Luttinger model with $J=\frac{3}{2}$ states, due to anisotropic backscattering off spin-polarized impurities aligned along the magnetic field. While a generalization to $\mathrm{Sr}_{3} \mathrm{SnO}$ with six copies of $J=\frac{3}{2}$ Dirac pockets is far from straightforward, if the same mechanism could produce competing AMR with a positive $C_{x x 2}$ contribution, it could explain the anomalous temperature dependence of $C_{x x 2}$ in our films. Furthermore, spin- $\frac{3}{2}$ states often manifest more anisotropy than spin- $\frac{1}{2}$ states, whether in the Rashba effect, where the spin expectation value winds three times instead of once in a $2 \pi$ rotation, or the Zeeman effect, where the spin splitting also modulates with the magnetic field direction $[49,50]$. Theoretical investigations of whether $J=\frac{3}{2}$ models can produce higher harmonics in the PHE, similar in methodology to Refs. [48,51], would shed much light.

In conclusion, we have detected sixfold oscillations in the PHE of the Dirac antiperovskite $\mathrm{Sr}_{3} \mathrm{SnO}$. Both the AMR and PHE are unusually complex, showing multiple harmonics with distinct behaviors with respect to magnetic field, temperature, and film thickness. Our results exemplify the richness of the PHE and motivate further investigations of higher harmonics in a wide range of topological materials. This paper also exposes the need for more theoretical studies to elucidate the microscopic driving forces.

\section{ACKNOWLEDGMENTS}

We thank Y.-h. Chan, M. Hirschmann, G. Jackeli, and A. Schnyder for useful discussions and M. Dueller, C. Mühle, K. Pflaum, and S. Schmid for technical support. D.H. acknowledges support from a Humboldt Research Fellowship for Postdoctoral Researchers.
[1] K. M. Koch, Notizen: Zum Problem der galvanomagnetischen Effekte in Ferromagneticis, Z. Naturforsch. A 10, 496 (1955).

[2] C. Goldberg and R. E. Davis, New galvanomagnetic effect, Phys. Rev. 94, 1121 (1954).

[3] A. A. Burkov, Giant planar Hall effect in topological metals, Phys. Rev. B 96, 041110(R) (2017).

[4] S. Nandy, G. Sharma, A. Taraphder, and S. Tewari, Chiral Anomaly as the Origin of the Planar Hall Effect in Weyl Semimetals, Phys. Rev. Lett. 119, 176804 (2017).

[5] H. Li, H.-W. Wang, H. He, J. Wang, and S.-Q. Shen, Giant anisotropic magnetoresistance and planar Hall effect in the Dirac semimetal $\mathrm{Cd}_{3} \mathrm{As}_{2}$, Phys. Rev. B 97, 201110(R) (2018).

[6] S. Liang, J. Lin, S. Kushwaha, J. Xing, N. Ni, R. J. Cava, and N. P. Ong, Experimental Tests of the Chiral Anomaly Magnetoresistance in the Dirac-Weyl Semimetals $\mathrm{Na}_{3} \mathrm{Bi}$ and GdPtBi, Phys. Rev. X 8, 031002 (2018).
[7] N. Kumar, S. N. Guin, C. Felser, and C. Shekhar, Planar Hall effect in the Weyl semimetal GdPtBi, Phys. Rev. B 98, 041103(R) (2018).

[8] F. C. Chen, X. Luo, J. Yan, Y. Sun, H. Y. Lv, W. J. Lu, C. Y. Xi, P. Tong, Z. G. Sheng, X. B. Zhu, W. H. Song, and Y. P. Sun, Planar Hall effect in the type-II Weyl semimetal $T_{d}-\mathrm{MoTe}_{2}$, Phys. Rev. B 98, 041114(R) (2018).

[9] R. Singha, S. Roy, A. Pariari, B. Satpati, and P. Mandal, Planar Hall effect in the type-II Dirac semimetal $\mathrm{VAl}_{3}$, Phys. Rev. B 98, 081103(R) (2018).

[10] P. Li, C. H. Zhang, J. W. Zhang, Y. Wen, and X. X. Zhang, Giant planar Hall effect in the Dirac semimetal $\mathrm{ZrTe}_{5-\delta}$, Phys. Rev. B 98, 121108(R) (2018).

[11] M. Wu, G. Zheng, W. Chu, Y. Liu, W. Gao, H. Zhang, J. Lu, Y. Han, J. Zhou, W. Ning, and M. Tian, Probing the chiral anomaly by planar Hall effect in Dirac semimetal $\mathrm{Cd}_{3} \mathrm{As}_{2}$ nanoplates, Phys. Rev. B 98, 161110(R) (2018). 
[12] J. Yang, W. L. Zhen, D. D. Liang, Y. J. Wang, X. Yan, S. R. Weng, J. R. Wang, W. Tong, L. Pi, W. K. Zhu, and C. J. Zhang, Current jetting distorted planar Hall effect in a Weyl semimetal with ultrahigh mobility, Phys. Rev. Mater. 3, 014201 (2019).

[13] Q. Liu, F. Fei, B. Chen, X. Bo, B. Wei, S. Zhang, M. Zhang, F. Xie, M. Naveed, X. Wan, F. Song, and B. Wang, Nontopological origin of the planar Hall effect in the type-II Dirac semimetal $\mathrm{NiTe}_{2}$, Phys. Rev. B 99, 155119 (2019).

[14] D. D. Liang, Y. J. Wang, W. L. Zhen, J. Yang, S. R. Weng, X. Yan, Y. Y. Han, W. Tong, W. K. Zhu, L. Pi, and C. J. Zhang, Origin of planar Hall effect in type-II Weyl semimetal $\mathrm{MoTe}_{2}$, AIP Adv. 9, 055015 (2019).

[15] J. Meng, H. Xue, M. Liu, W. Jiang, Z. Zhang, J. Ling, L. He, R. Dou, C. Xiong, and J. Nie, Planar Hall effect induced by anisotropic orbital magnetoresistance in type-II Dirac semimetal PdTe 2 , J. Phys.: Condens. Matter 32, 015702 (2019).

[16] P. Li, C. Zhang, Y. Wen, L. Cheng, G. Nichols, D. G. Cory, G.-X. Miao, and X.-X. Zhang, Anisotropic planar Hall effect in the type-II topological Weyl semimetal $\mathrm{WTe}_{2}$, Phys. Rev. B 100, 205128 (2019).

[17] Z. Li, T. Xiao, R. Zou, J. Li, Y. Zhang, Y. Zeng, M. Zhou, J. Zhang, and W. Wu, Planar Hall effect in $\mathrm{PtSe}_{2}$, J. Appl. Phys. 127, 054306 (2020)

[18] S.-Y. Yang, K. Chang, and S. S. P. Parkin, Large planar Hall effect in bismuth thin films, Phys. Rev. Research 2, 022029(R) (2020).

[19] A. A. Taskin, H. F. Legg, F. Yang, S. Sasaki, Y. Kanai, K. Matsumoto, A. Rosch, and Y. Ando, Planar Hall effect from the surface of topological insulators, Nat. Commun. 8, 1340 (2017).

[20] M. Shaviv Petrushevsky, P. K. Rout, G. Levi, A. Kohn, and Y. Dagan, Signature of surface state coupling in thin films of the topological Kondo insulator $\mathrm{SmB}_{6}$ from anisotropic magnetoresistance, Phys. Rev. B 95, 085112 (2017).

[21] L. Zhou, B. C. Ye, H. B. Gan, J. Y. Tang, P. B. Chen, Z. Z. Du, Y. Tian, S. Z. Deng, G. P. Guo, H. Z. Lu, F. Liu, and H. T. $\mathrm{He}$, Surface-induced positive planar Hall effect in topological Kondo insulator $\mathrm{SmB}_{6}$ microribbons, Phys. Rev. B 99, 155424 (2019).

[22] P. K. Rout, I. Agireen, E. Maniv, M. Goldstein, and Y. Dagan, Six-fold crystalline anisotropic magnetoresistance in the (111) $\mathrm{LaAlO}_{3} / \mathrm{SrTiO}_{3}$ oxide interface, Phys. Rev. B 95, 241107(R) (2017).

[23] J. H. Ngai, Y. Segal, F. J. Walker, and C. H. Ahn, Electrostatic modulation of anisotropic magnetotransport in $\mathrm{Ar}^{+}$-irradiated $\mathrm{SrTiO}_{3}$ : effects of boundary scattering, Phys. Rev. B 83, 045304 (2011).

[24] A. Annadi, Z. Huang, K. Gopinadhan, X. R. Wang, A. Srivastava, Z. Q. Liu, H. H. Ma, T. P. Sarkar, T. Venkatesan, and Ariando, Fourfold oscillation in anisotropic magnetoresistance and planar Hall effect at the $\mathrm{LaAlO}_{3} / \mathrm{SrTiO}_{3}$ heterointerfaces: effect of carrier confinement and electric field on magnetic interactions, Phys. Rev. B 87, 201102(R) (2013).

[25] L. Miao, R. Du, Y. Yin, and Q. Li, Anisotropic magnetotransport properties of electron gases at $\mathrm{SrTiO}_{3}$ (111) and (110) surfaces, Appl. Phys. Lett. 109, 261604 (2016).

[26] H. J. Harsan Ma, J. Zhou, M. Yang, Y. Liu, S. W. Zeng, W. X. Zhou, L. C. Zhang, T. Venkatesan, Y. P. Feng, and Ariando, Giant crystalline anisotropic magnetoresistance in nonmagnetic perovskite oxide heterostructures, Phys. Rev. B 95, 155314 (2017).
[27] N. Wadehra, R. Tomar, R. M. Varma, R. K. Gopal, Y. Singh, S. Dattagupta, and S. Chakraverty, Planar Hall effect and anisotropic magnetoresistance in polar-polar interface of $\mathrm{LaVO}_{3}-\mathrm{KTaO}_{3}$ with strong spin-orbit coupling, Nat. Commun. 11, 874 (2020).

[28] T. H. Hsieh, J. Liu, and L. Fu, Topological crystalline insulators and Dirac octets in antiperovskites, Phys. Rev. B 90, 081112(R) (2014).

[29] T. Kariyado and M. Ogata, Three-dimensional Dirac electrons at the Fermi energy in cubic inverse perovskites: $\mathrm{Ca}_{3} \mathrm{PbO}$ and its family, J. Phys. Soc. Jpn. 80, 083704 (2011).

[30] T. Kariyado and M. Ogata, Low-energy effective Hamiltonian and the surface states of $\mathrm{Ca}_{3} \mathrm{PbO}$, J. Phys. Soc. Jpn. 81, 064701 (2012).

[31] T. Kariyado and M. Ogata, Evolution of band topology by competing band overlap and spin-orbit coupling: twin Dirac cones in $\mathrm{Ba}_{3} \mathrm{SnO}$ as a prototype, Phys. Rev. Mater. 1, 061201(R) (2017).

[32] C.-K. Chiu, Y.-H. Chan, X. Li, Y. Nohara, and A. P. Schnyder, Type-II Dirac surface states in topological crystalline insulators, Phys. Rev. B 95, 035151 (2017).

[33] Y. Fang and J. Cano, Higher-order topological insulators in antiperovskites, Phys. Rev. B 101, 245110 (2020).

[34] S. Kitagawa, K. Ishida, M. Oudah, J. N. Hausmann, A. Ikeda, S. Yonezawa, and Y. Maeno, Normal-state properties of the antiperovskite oxide $\mathrm{Sr}_{3-x} \mathrm{SnO}$ revealed by ${ }^{119} \mathrm{Sn}-\mathrm{NMR}$, Phys. Rev. B 98, 100503(R) (2018).

[35] A. W. Rost, J. Kim, S. Suetsugu, V. Abdolazimi, K. Hayama, J. A. N. Bruin, C. Mühle, K. Kitagawa, A. Yaresko, J. Nuss, and $\mathrm{H}$. Takagi, Inverse-perovskites $A_{3} B \mathrm{O}(A=\mathrm{Sr}, \mathrm{Ca}, \mathrm{Eu} / B=$ $\mathrm{Pb}, \mathrm{Sn})$ : A platform for control of Dirac and Weyl Fermions, APL Mater. 7, 121114 (2019).

[36] Y. Ma, A. Edgeton, H. Paik, B. D. Faeth, C. T. Parzyck, B. Pamuk, S.-L. Shang, Z.-K. Liu, K. M. Shen, D. G. Schlom, and C.-B. Eom, Realization of epitaxial thin films of the topological crystalline insulator $\mathrm{Sr}_{3} \mathrm{SnO}$, Adv. Mater. 32, 2000809 (2020).

[37] M. Oudah, A. Ikeda, J. N. Hausmann, S. Yonezawa, T. Fukumoto, S. Kobayashi, M. Sato, and Y. Maeno, Superconductivity in the antiperovskite Dirac-metal oxide $\mathrm{Sr}_{3-x} \mathrm{SnO}$, Nat. Commun. 7, 13617 (2016).

[38] T. Kawakami, T. Okamura, S. Kobayashi, and M. Sato, Topological Crystalline Materials of $J=3 / 2$ Electrons: Antiperovskites, Dirac Points, and High Winding Topological Superconductivity, Phys. Rev. X 8, 041026 (2018).

[39] H. Nakamura, D. Huang, J. Merz, E. Khalaf, P. Ostrovsky, A Yaresko, D. Samal, and H. Takagi, Robust weak antilocalization due to spin-orbital entanglement in Dirac material $\mathrm{Sr}_{3} \mathrm{SnO}$, Nat. Commun. 11, 1161 (2020).

[40] D. Huang, H. Nakamura, K. Küster, A. Yaresko, D. Samal, N. B. M. Schröter, V. N. Strocov, U. Starke, and H. Takagi, Unusual valence state in the antiperovskites $\mathrm{Sr}_{3} \mathrm{SnO}$ and $\mathrm{Sr}_{3} \mathrm{PbO}$ revealed by $\mathrm{x}$-ray photoelectron spectroscopy, Phys. Rev. Mater. 3, 124203 (2019).

[41] K. Momma and F. Izumi, VESTA3 for three-dimensional visualization of crystal, volumetric and morphology data, J. Appl. Cryst. 44, 1272 (2011).

[42] See Supplemental Material at http://link.aps.org/supplemental/ 10.1103/PhysRevResearch.3.013268 for further information regarding effects due to an out-of-plane magnetic field and additional data on 50-nm-thick films. 
[43] S.-H. Zheng, H.-J. Duan, J.-K. Wang, J.-Y. Li, M.-X. Deng, and R.-Q. Wang, Origin of planar Hall effect on the surface of topological insulators: tilt of Dirac cone by an in-plane magnetic field, Phys. Rev. B 101, 041408(R) (2020).

[44] A. W. Rushforth, K. Výborný, C. S. King, K. W. Edmonds, R. P. Campion, C. T. Foxon, J. Wunderlich, A. C. Irvine, P. Vašek, V. Novák, K. Olejník, J. Sinova, T. Jungwirth, and B. L. Gallagher, Anisotropic Magnetoresistance Components in (Ga,Mn)As, Phys. Rev. Lett. 99, 147207 (2007).

[45] R. Xu, A. Husmann, T. F. Rosenbaum, M. L. Saboungi, J. E. Enderby, and P. B. Littlewood, Large magnetoresistance in nonmagnetic silver chalcogenides, Nature 390, 57 (1997).

[46] A. L. Friedman, J. L. Tedesco, P. M. Campbell, J. C. Culbertson, E. Aifer, F. K. Perkins, R. L. Myers-Ward, J. K. Hite, C. R. Eddy, G. G. Jernigan, and D. K. Gaskill, Quantum linear magnetoresistance in multilayer epitaxial graphene, Nano Lett. 10, 3962 (2010)
[47] X. Wang, Y. Du, S. Dou, and C. Zhang, Room Temperature Giant and Linear Magnetoresistance in Topological Insulator $\mathrm{Bi}_{2} \mathrm{Te}_{3}$ Nanosheets, Phys. Rev. Lett. 108, 266806 (2012).

[48] M. Trushin, K. Výborný, P. Moraczewski, A. A. Kovalev, J. Schliemann, and T. Jungwirth, Anisotropic magnetoresistance of spin-orbit coupled carriers scattered from polarized magnetic impurities, Phys. Rev. B 80, 134405 (2009).

[49] R. Winkler, D. Culcer, S. J. Papadakis, B. Habib, and M. Shayegan, Spin orientation of holes in quantum wells, Semicond. Sci. Technol. 23, 114017 (2008).

[50] H. Nakamura, T. Koga, and T. Kimura, Experimental Evidence of Cubic Rashba Effect in an Inversion-Symmetric Oxide, Phys. Rev. Lett. 108, 206601 (2012).

[51] N. Boudjada, I. Khait, and A. Paramekanti, Anisotropic magnetoresistance in multiband systems: Two-dimensional electron gases and polar metals at oxide interfaces, Phys. Rev. B 99, 195453 (2019) 